\title{
Monitoring method for carbon nanotubes (CNT): Personal sampler and corresponding reading device
}

\author{
M. Keller ${ }^{a}$, G. Kreck ${ }^{a}$, Y. Holzapfel ${ }^{a}$, N. Neubauer ${ }^{b}$, M. Seipenbusch ${ }^{b}$ \\ ${ }^{a}$ Fraunhofer Institute for Manufacturing Engineering and Automation IPA, 70569 Stuttgart, Germany \\ ${ }^{b}$ Karlsruhe Institut für Technologie KIT, 76131 Karlsruhe, Germany
}

\begin{abstract}
At present, CNT and other nanofiber materials in air can only be detected by deposition on a substrate and the use of off-line imaging analysis such as scanning electron microscopy. The majority of techniques suitable for the quasi-realtime measurement of engineered nanoparticles such as electrical low pressure impactor, condensation particle counter, optionally combined with a scanning mobility particle sizer (SMPS) can not distinguish between nanoparticles in general and airborne nanofibres in particular. In common workplace settings the considerable background of fine and ultrafine particles thus poses a challenge for these instruments.

Furthermore, a true portability of the present devices is not given due to their size and power consumption. However, there is an urgent need due to the expected toxicity of nanofibres to control workplace environments with a robust and mobile device to ensure the safety of the working personnel as soon as possible.

To meet these challenges, a suitable personal sampler together with a corresponding reading device is under development in the NANODEVICE-project, funded by the European Commission under grant agreement FP7-2114642. A pre-prototype personal sampler for CNT sampling, the used Raman-spectroscopic inspection of deposited CNTs and a possible approach in determining the qualitative presence of CNTs will be shown. Additionally, it seems even possible to distinguish between carbon nanofibers from different manufacturers based on Raman spectroscopy and data analysis.
\end{abstract}

Keywords: CNT, nanofiber, workplace safety, personal sampler, Raman-spectroscopy, NANODEVICE, NanoSafety

\section{Introduction}

At present, carbon nanotubes (CNT) are industrially produced in tons by several production plants overall the globe. CNTs show unique electrical, mechanical and thermal properties and have many potential applications in the electronic, plastic, computer and aerospace industries [1]. CNTs are regarded as potentially harmful substabces and many research topics deal with the toxicity of CNTs.

Accidental release of CNTs into the air can in principal harm workers after inhaling the contaminated air as aerosol. Thus, it is necessary to monitor these risks areas to trigger an alarm if a prior set limit is exceeded. For this purpose, a simple personal sampler shall monitor selected environments about the accidental release of CNTs in the near future.

For the development of such a personal fiber monitor, a two-stage approach is conducted: a) The personal sampler will collect CNTs on an appropriate substrate and b) the substrate is analyzed in a laboratory setting using a device with a high potential for miniaturization.

\section{Material and Methods}

\subsection{CNT agglomeration}

To demonstrate the general agglomeration status of deposited CNTs, the following setup was selected: After CNT-generation at the KIT using a chemical vapour deposition process (CVD-process), the CNTs were deposited by impaction on silicon wafer (Fig. 1) for later analysis using scanning electron microscopy (SEM) and grids for later analysis using transmission electron microscopy (TEM).

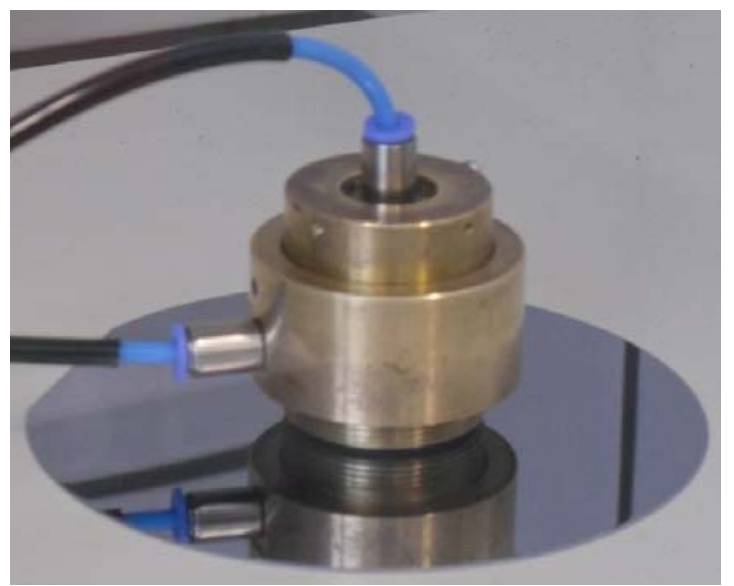

Fig. 1. Sampling CNTs directly on silicon wafer 


\subsection{Personal sampler}

The newly designed pre-prototype personal sampler collects all kind of particles on a suitable substrate (Fig. 2). Using a combination of impaction (removal of larger particles) and electrostatic deposition (collection of highly charged particles as CNTs) enables a controlled concentration of the particles of interest on a very small spot for later analysis.

The personal sampler uses a battery-driven sampling pump (SG 350, GSA Messgerätebau Neuss $\mathrm{GmbH}$ ) with the opportunity to fix a sampling time and sampling flow rate (Fig 1a-1). The pump is connected to the sampler with a flexible silicone tubing (Fig 2a-2). The housing of the sampler consists of a commercially available box made of glass fibre reinforced polycarbonate (Fig 2a-3 and $2 \mathrm{~b}-3$ ). The used straight stainless steel tubing for sampling has an inner tube diameter of $1 \mathrm{~mm}$ and a length of $0.15 \mathrm{~m}$ (Fig 2a-4. Note: In Fig. 2a, a coiled tube is shown)

As substrate, commercially available glass slides are selected due to smooth surface needed for later analysis, availability and price (Fig. 2b-4). Further improvement will include a small electrode to collect highly charged particles as CNTs on a defined spot.

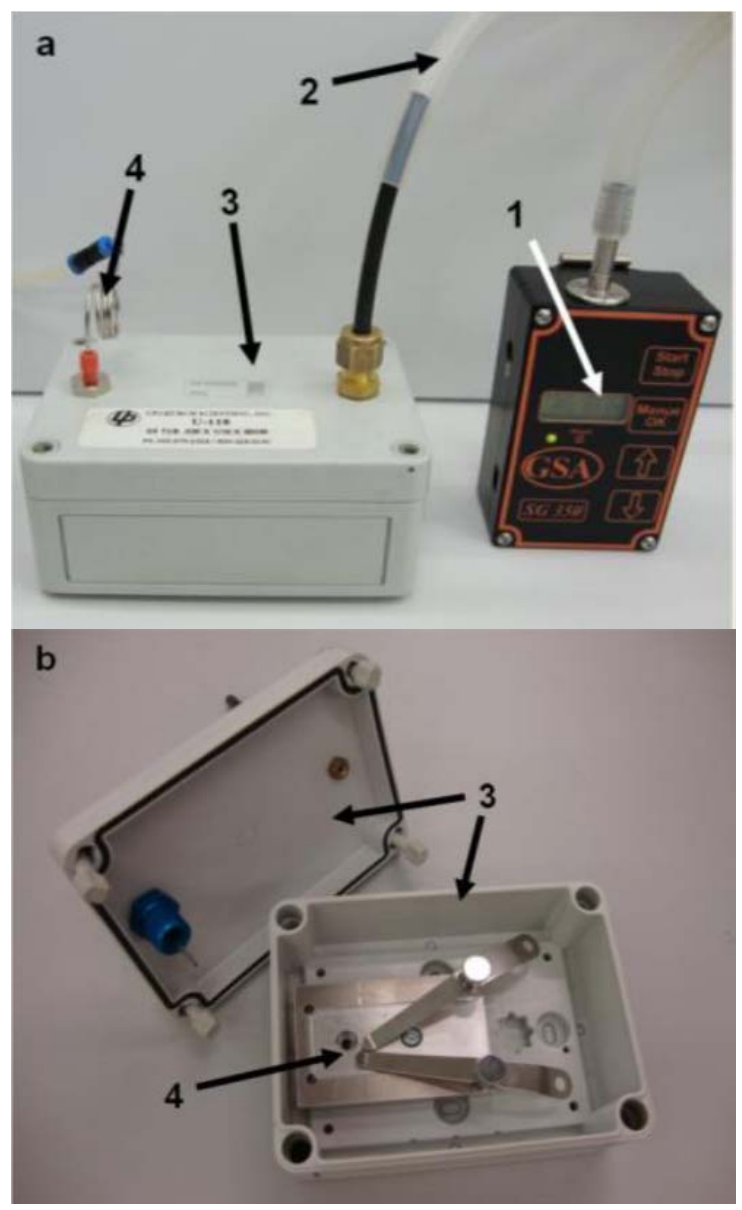

Fig. 2. Pre-prototype of a personal sampler.

\subsection{1.} Lab-scale CNT sampling using the personal sampler

The personal sampler was equipped with a precleaned glass slide. The closed housing was fitted to a personal sampling pump with a flow rate of $100 \mathrm{ml} / \mathrm{min}$. The inlet was placed directly after the outlet of the CVDgenerator for five minutes.

The glass slide was examined using reflected light microscopy with automated picture stitching (Olympus BX 51 combined with Olympus particle inspector software). The $5 x$ objective with a resolution of $2.2 \mu \mathrm{m}$ was used, so smaller particles cannot be detected.

\subsubsection{Real-scenario sampling using the personal sampler}

The personal sampler was equipped with a precleaned glass slide. The closed housing was fitted to a personal sampling pump with a flow rate of $100 \mathrm{ml} / \mathrm{min}$. The whole setup was placed in a real scenario for handling CNTs. The shown sample was taken at an industrial setting by the Institute for Occupational Safety and Health of the German Social Accident Insurance (IFA) for 42 minutes. Estimated particulate contamination can be polymer-particles with attached CNTs, carbon black and other carbon-particulate matter and boundless CNT agglomerates and single CNT fibers.

The glass slide was examined using the inVia Raman microscope from Renishaw $\mathrm{GmbH}$ with an excitation wave length of $532 \mathrm{~nm}$ and the data acquisition automation StreamLineHR.

\subsection{Raman analysis of CNT agglomerates}

Raman spectroscopy is a spectroscopic technique used to study vibrational, rotational, and other lowfrequency modes in a system. It relies on inelastic scattering of monochromatic light. The monochromatic light interacts with molecular vibrations, phonons or other excitations in the system, resulting in the energy of the laser photons being shifted up or down. The shift in energy gives information about the phonon modes in the system [3]. In principal, Raman spectroscopy has a high potential in miniaturization.

For first qualitative analysis, a small amount of commercially available multi-walled mwCNTs (Baytubes C 150 HP, Bayer MaterialScience AG, Leverkusen, Germany; NC 7000, Nanocyl s.a., Sambreville, Belgium; $C_{\text {Tube }}$ 200, CNT Co., Yeonsu-Gu, Korea and ATI MWCNT-001, Ahwahnee Technology Inc., San Jose, United States) were used. The mw-CNT powders were put manually onto a gold-coated surface. All samples were inspected using a) the Raman-based single particle explorer system from Rap-ID particle systems $\mathrm{GmbH}$, Berlin, Germany; b) Raman-Station 400 coupled with Raman Micro 300 from PerkinElmer Inc., Waltham, United States and inVia Raman 
microscope, Renishaw plc, Gloucestershire, United Kingdom. The obtained data (spectra) were transferred into the existing databases from each manufacturer for later spectra comparison. After the compilation of the obtained spectra into an existing database, all mentioned carbon-containing materials were analyzed randomly.

\section{Results}

\subsection{CNT agglomeration}

The SEM (Fig. 3) and TEM-analysis (Fig. 4) of the general CNT agglomeration status of deposited CNTs after chemical vapour deposition process showed the following results:

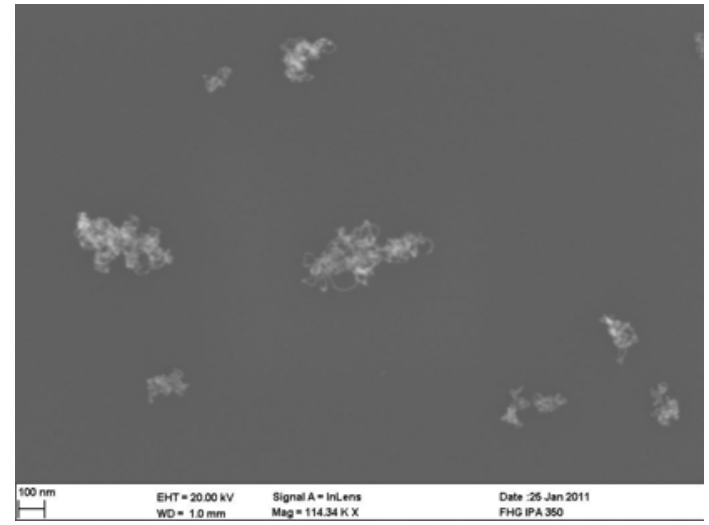

Fig. 1. SEM-analysis of CNTs deposited on silicon surface. The measurement bar indicates $100 \mathrm{~nm}$

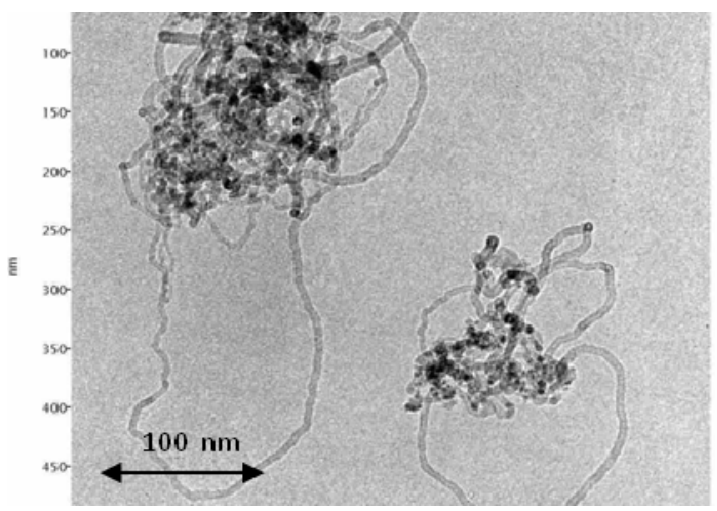

Fig. 2. TEM-analysis of CNTs deposited on a TEMgrid. The measurement bar indicates $100 \mathrm{~nm}$

Both approaches demonstrated that most agglomerates are larger than $100 \mathrm{~nm}$. It is assumed that by elongation of the sampling duration, secondary agglomerates are generated directly on the substrate. These secondary agglomerates facilitate the later applied analysis using RAMAN-spectroscopy.

\subsection{Lab-scale CNT sampling using the personal sampler}

By sampling CNTs directly after CVD-generation, following deposition pattern was visible [2]. The characteristic deposition beam to the right of Fig. 5 was visible by all CNT sampling approaches, but never by only sampling background aerosol (e.g. soot, ambient atmosphere). It is assumed that the ESD characteristic of the used plastic housing plays the major role in generating this deposition pattern. For the confirmation of this hypothesis, a small electrode will be placed into the chamber to force charged particles which were not deposited by impaction due to their small size to this defined spot. This research is ongoing and the results will be shown at the $4 \mathrm{M}$ conference 2011 .

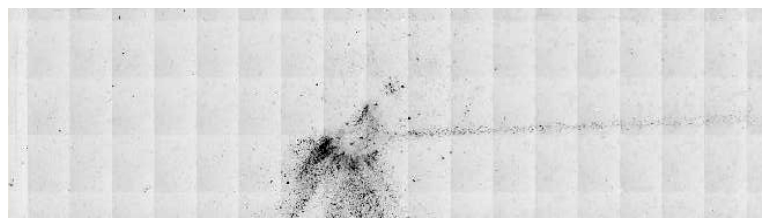

Fig. 3. Deposition pattern and collected background and CNT particles

\subsection{Real-scenario sampling using the personal sampler}

Using the inVia Raman microscope from Renishaw $\mathrm{GmbH}$ with an excitation wave length of $532 \mathrm{~nm}$, a 100x lens and the data acquisition automation StreamLineHR, a surface of $16 \times 14 \mu \mathrm{m}$ from the sample obtained from chapter 2.2.2 was analyzed with a step width of $0,5 \mu \mathrm{m}$. Therefore, 957 individual spectra were combined to a topographical spectra map. Based on this topographical spectra map, different analysis algorithms were performed. The main component analysis scans the whole map and compares the individual spectra with spectra present in a database available at Renishaw. Two different major components of the analyzed particles could be detected (Fig. 6). Component 1 was classified as charcoal. Component 2 was not possible to classify to a specific material present in the database.

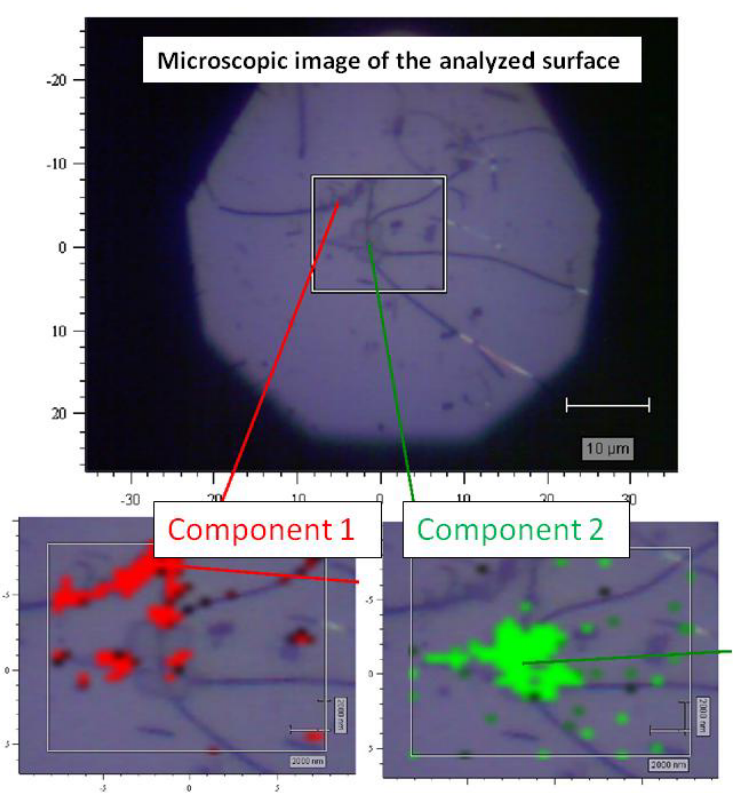


Fig. 6. Microscopic image and overlay of the Ramanimage after major component analysis of the analyzed surface

\subsection{Qualitative Raman-analysis - spectra}

\subsubsection{Single particle explorer, Rap-ID particle} systems $\mathrm{GmbH}$

Using the single particle inspection system SPE CSS from Rap-ID with an excitation wave length of 532 $\mathrm{nm}$, the following spectra were obtained, see following Fig. 7. The $x$-axis represents the Raman-shift $[1 / \mathrm{cm}]$ and the $y$-axis the measured relative intensity. Ten different agglomerates were measured and the mean value of all ten measurements is shown in black. This mean value was read into the database for later spectra comparison, see chapter 3.4 .
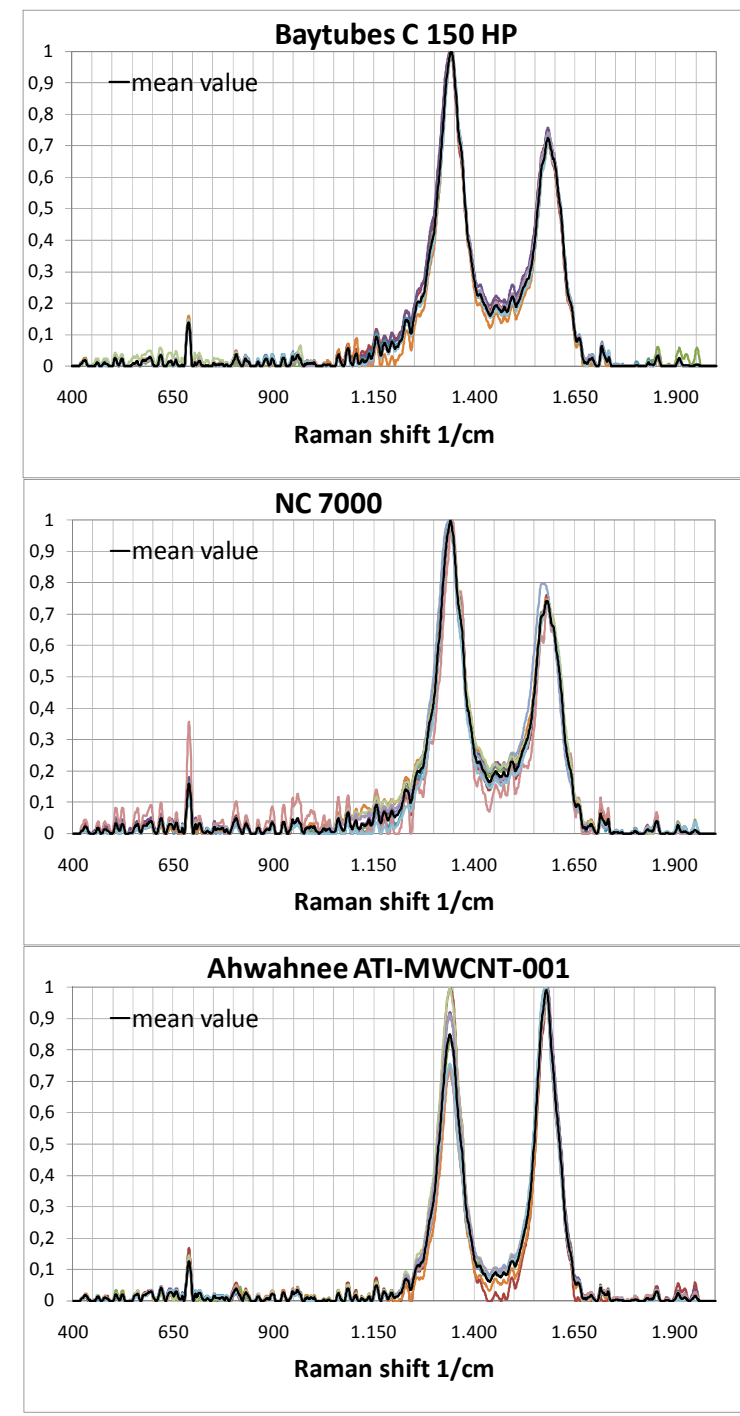
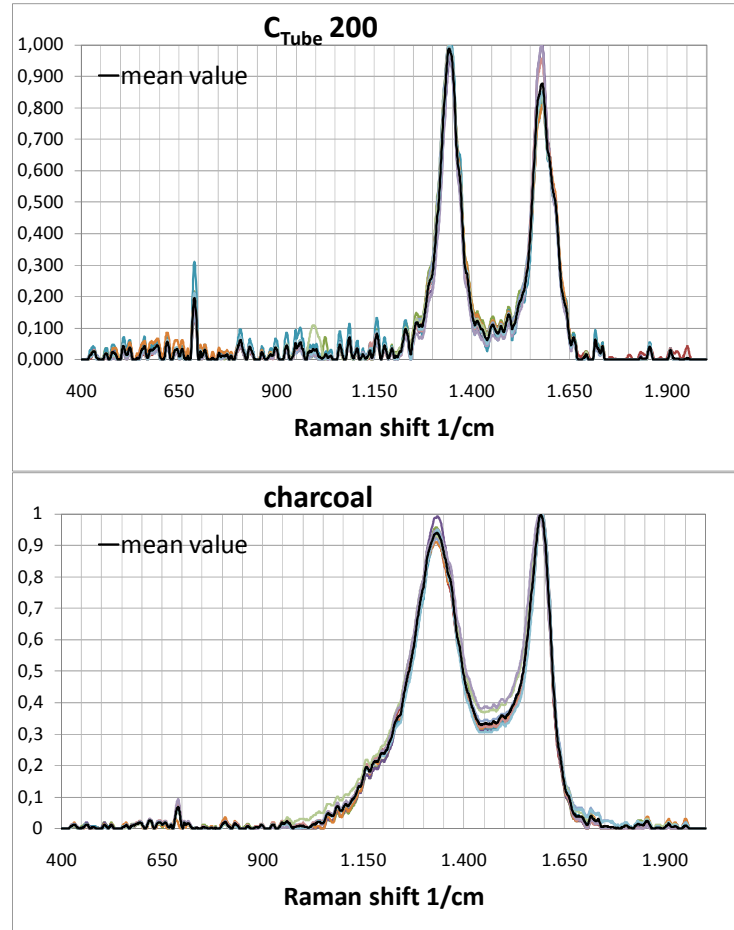

Fig. 7. Raman-spectrum of a) Baytubes $150 \mathrm{HP}$ (top); b) Nanocyl NC 7000 (upper middle) Ahwahnee MWCNT-001 (middle), $\mathrm{C}_{\text {Tube }} 200$ (lower middle) and charcoal (bottom)

\subsubsection{Raman-Station 400, PerkinElmer LAS GmbH}

Using the Raman-Station 400 coupled with Raman Micro 300 with an excitation wave length of $785 \mathrm{~nm}$ from PerkinElmer LAS $\mathrm{GmbH}$, the following spectra were obtained, see following Fig. 8. The x-axis represents the Raman-shift $[1 / \mathrm{cm}]$ and the $y$-axis the measured relative intensity.

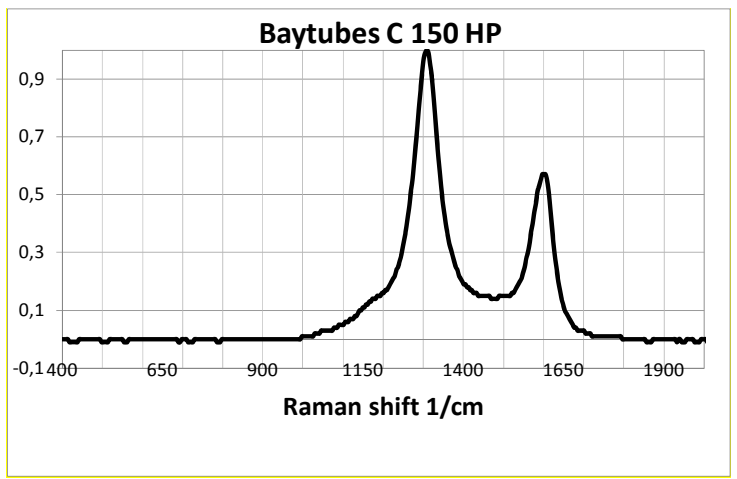




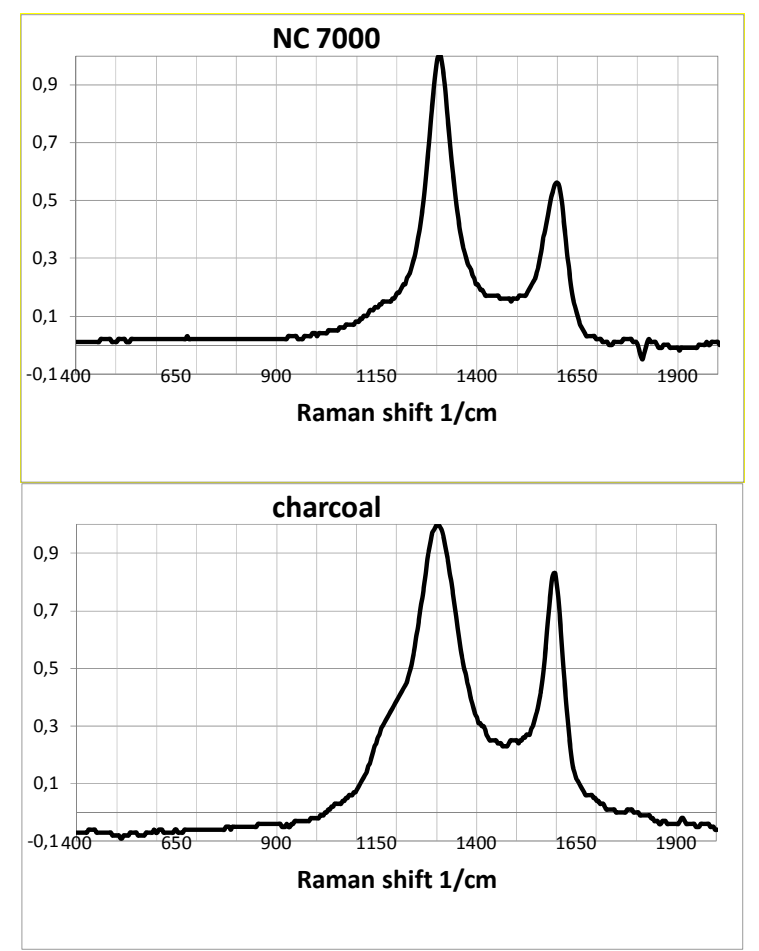

Fig. 8. Raman-spectrum of a) Baytubes $150 \mathrm{HP}$ (top); b) Nanocyl NC 7000 (middle) and charcoal (bottom)

\subsection{3. inVia Raman microscope, Renishaw $\mathrm{GmbH}$}

Using the inVia Raman microscope from Renishaw $\mathrm{GmbH}$ with an excitation wave length of 532 $\mathrm{nm}$, the following spectra were obtained, see following Fig. 9. The $x$-axis represents the Raman-shift $[1 / \mathrm{cm}]$ and the $y$-axis the measured relative intensity.

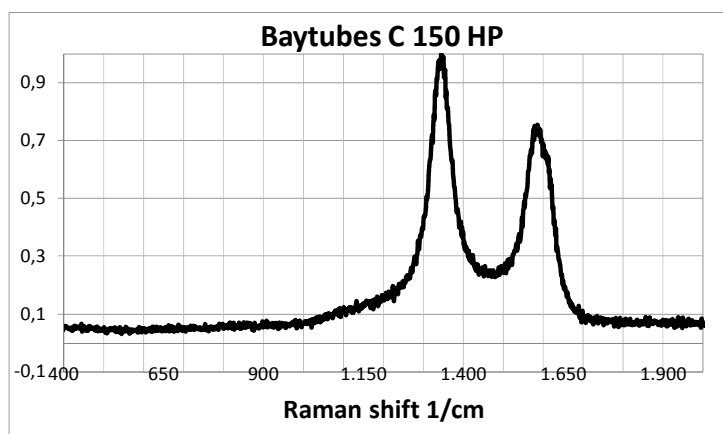

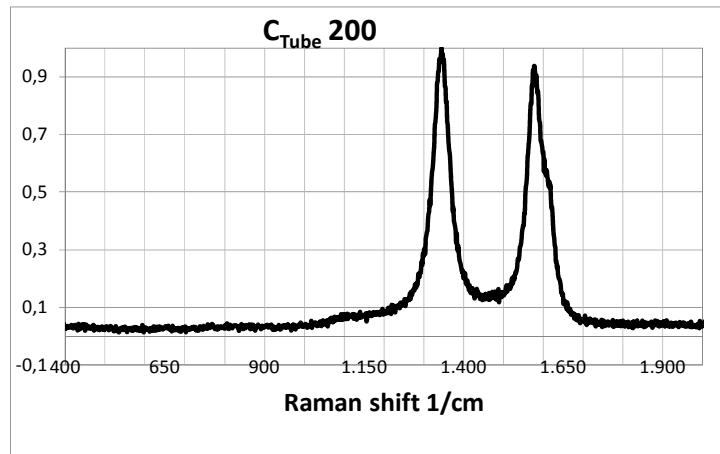
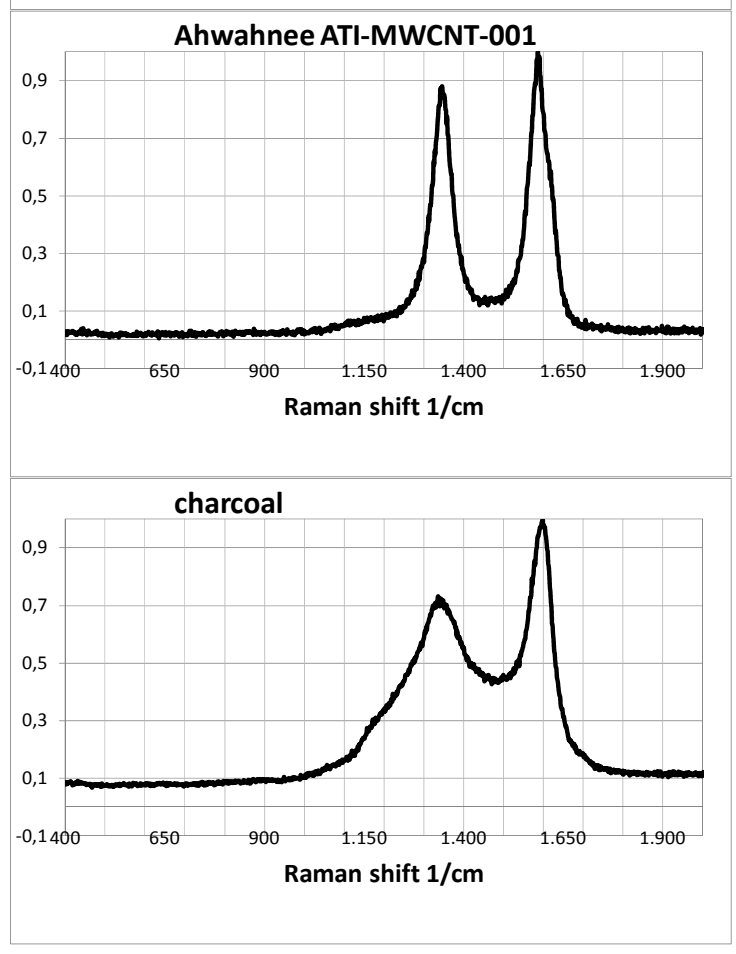

Fig. 9. Raman-spectrum of a) Baytubes $150 \mathrm{HP}$ (top); b) $\mathrm{C}_{\text {Tube }} 200$ (upper middle); Ahwahnee MWCNT-001 (lower middle) and charcoal (bottom)

The sample Nanocyl NC 7000 was measured with three different excitation wavelengths of $\lambda_{1}=532$ $\mathrm{nm}, \lambda_{2}=633 \mathrm{~nm}$ and $\lambda_{3}=785 \mathrm{~nm}$ and an extended Raman shift scan range from 100 to $3200 \mathrm{~cm}^{-1}$. The result is shown in Fig. 10 and in detail in Fig. 11.

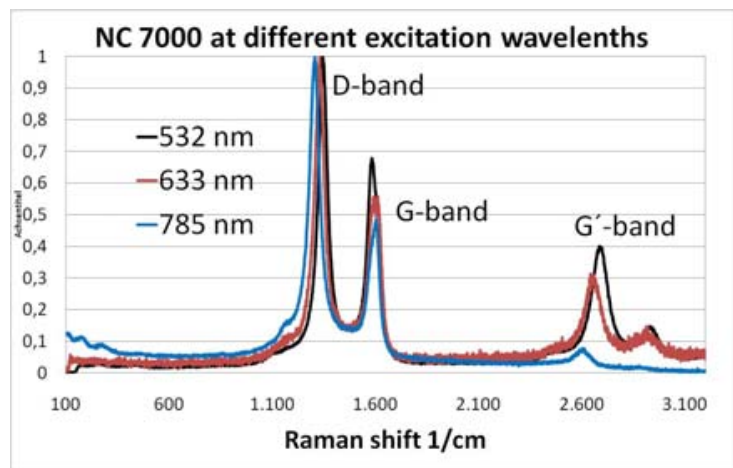

Fig. 10. Raman-spectrum of NC 7000 at different 
excitation wavelengths

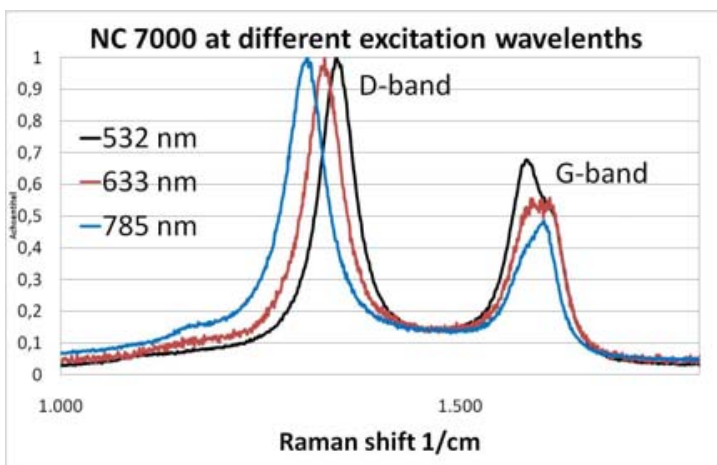

Fig. 11. Raman-spectrum of NC 7000 at different excitation wavelengths -detail

It is clearly visible that the Raman-shift of the peaks of the D-band and G'-band decreases with increasing excitation wavelength, whereas the peaks of the G-band increase with increasing excitation wavelength.

\subsection{Qualitative Raman analysis - database comparison}

Using the single particle inspection system SPE CSS from Rap-ID $\mathrm{GmbH}$, its database for spectra comparison, the imported ten spectra from each mwCNT-source (see chapter 3.3.1) and several analyzed spectra from other carbon-based surfaces and materials (graphite, charcoal, diamond, diamondlike carbon (DLC) surfaces as $\mathrm{a}-\mathrm{CH}$, ta-C and ta-CCF4) were used. Therefore, the powders were put manually onto a gold-coated surface and the DLCsurfaces were inspected directly

The used algorithm for comparison was the Pearson correlation algorithm [4]. Only hit 1 to 3 with the name and correlation factor is shown due to illustration purposes. The correlation factor of a non-mwCNT source was always below $95 \%$.

\begin{tabular}{|c|c|c|c|c|c|c|}
\hline Ahwahnee & \multicolumn{2}{|c|}{ Hit 1 } & \multicolumn{2}{c|}{ Hit 2 } & \multicolumn{2}{c|}{ Hit 3 } \\
\hline Sample ID & Result: & $\mathbf{\%}$ & Result: & \% & Result: & \% \\
\hline 1 & Ahwahnee & $\mathbf{9 9 . 8}$ & Ahwahnee & $\mathbf{9 8 . 6}$ & Ahwahnee & $\mathbf{9 8 . 1}$ \\
\hline 2 & Ahwahnee & $\mathbf{9 9 . 2}$ & Ahwahnee & $\mathbf{9 9 . 2}$ & C-Tubes 200 & $\mathbf{9 9 . 1}$ \\
\hline 3 & Ahwahnee & $\mathbf{9 9 . 2}$ & Ahwahnee & $\mathbf{9 9 . 1}$ & C-Tubes 200 & $\mathbf{9 9 . 1}$ \\
\hline 4 & Ahwahnee & $\mathbf{9 9 . 8}$ & Ahwahnee & $\mathbf{9 9 . 6}$ & Ahwahnee & $\mathbf{9 9 . 5}$ \\
\hline 5 & Ahwahnee & $\mathbf{9 9 . 8}$ & Ahwahnee & $\mathbf{9 9 . 7}$ & Ahwahnee & $\mathbf{9 9 . 7}$ \\
\hline 6 & Ahwahnee & $\mathbf{9 9 . 7}$ & Ahwahnee & $\mathbf{9 9 . 6}$ & Ahwahnee & $\mathbf{9 9 . 5}$ \\
\hline 7 & Ahwahnee & $\mathbf{9 9 . 5}$ & Ahwahnee & $\mathbf{9 9 . 5}$ & Ahwahnee & $\mathbf{9 9 . 5}$ \\
\hline 8 & Ahwahnee & $\mathbf{9 9 . 4}$ & C-Tubes 200 & $\mathbf{9 8 . 8}$ & C-Tubes 200 & $\mathbf{9 8 . 8}$ \\
\hline 9 & Ahwahnee & $\mathbf{9 9 . 5}$ & Ahwahnee & $\mathbf{9 8 . 8}$ & Ahwahnee & $\mathbf{9 8 . 7}$ \\
\hline 10 & Ahwahnee & $\mathbf{9 9 . 7}$ & Ahwahnee & $\mathbf{9 9 . 7}$ & Ahwahnee & $\mathbf{9 9 . 6}$ \\
\hline
\end{tabular}

Table 1. Results from comparison of spectra from ATI MWCNT-001 with references from the extended spectra database

\begin{tabular}{|c|c|c|c|c|c|c|}
\hline NC 7000 & \multicolumn{2}{|c|}{ Hit 1 } & \multicolumn{2}{c|}{ Hit 2 } & \multicolumn{2}{c|}{ Hit 3 } \\
\hline Sample ID & Result: & $\mathbf{\%}$ & Result: & $\mathbf{\%}$ & Result: & $\%$ \\
\hline 1 & NC 7000 & $\mathbf{9 9 . 7}$ & Baytubes & $\mathbf{9 9 . 6}$ & Baytubes & $\mathbf{9 9 . 6}$ \\
\hline 2 & Baytubes & $\mathbf{9 9 . 1}$ & Baytubes & $\mathbf{9 9 . 1}$ & Baytubes & $\mathbf{9 9 . 0}$ \\
\hline 3 & NC 7000 & $\mathbf{9 9 . 5}$ & Baytubes & $\mathbf{9 9 . 4}$ & Baytubes & $\mathbf{9 9 . 4}$ \\
\hline 4 & Baytubes & $\mathbf{9 9 . 6}$ & Baytubes & $\mathbf{9 9 . 6}$ & Baytubes & $\mathbf{9 9 . 6}$ \\
\hline 5 & NC 7000 & $\mathbf{9 9 . 7}$ & Baytubes & $\mathbf{9 9 . 6}$ & Baytubes & $\mathbf{9 9 . 6}$ \\
\hline 6 & Baytubes & $\mathbf{9 9 . 8}$ & Baytubes & $\mathbf{9 9 . 8}$ & NC 7000 & $\mathbf{9 9 . 8}$ \\
\hline 7 & NC 7000 & $\mathbf{9 9 . 8}$ & Baytubes & $\mathbf{9 9 . 7}$ & Baytubes & $\mathbf{9 9 . 7}$ \\
\hline 8 & Baytubes & $\mathbf{9 9 . 5}$ & NC 7000 & $\mathbf{9 9 . 5}$ & Baytubes & $\mathbf{9 9 . 4}$ \\
\hline 9 & Baytubes & $\mathbf{9 9 . 8}$ & Baytubes & $\mathbf{9 9 . 8}$ & NC 7000 & $\mathbf{9 9 . 8}$ \\
\hline 10 & Baytubes & $\mathbf{9 9 . 2}$ & Baytubes & $\mathbf{9 8 . 9}$ & C-Tubes 200 & $\mathbf{9 8 . 9}$ \\
\hline
\end{tabular}

Table 2. Results from comparison of spectra from NC 7000 mwCNT with references from the extended spectra database

\begin{tabular}{|c|c|c|c|c|c|c|}
\hline Baytubes & \multicolumn{2}{|c|}{ Hit 1 } & \multicolumn{2}{c|}{ Hit 2 } & \multicolumn{2}{c|}{ Hit 3 } \\
\hline Sample ID & Result: & $\mathbf{\%}$ & Result: & \% & Result: & \% \\
\hline 1 & NC 7000 & $\mathbf{9 9 . 4}$ & Baytubes & $\mathbf{9 8 . 5}$ & NC 7000 & $\mathbf{9 8 . 2}$ \\
\hline 2 & Baytubes & $\mathbf{9 9 . 4}$ & Baytubes & $\mathbf{9 9 . 2}$ & Baytubes & $\mathbf{9 9 . 2}$ \\
\hline 3 & Baytubes & $\mathbf{9 9 . 8}$ & Baytubes & $\mathbf{9 9 . 6}$ & NC 7000 & $\mathbf{9 9 . 6}$ \\
\hline 4 & NC 7000 & $\mathbf{9 9 . 3}$ & Baytubes & $\mathbf{9 9 . 1}$ & Baytubes & $\mathbf{9 9 . 1}$ \\
\hline 5 & Baytubes & $\mathbf{9 9 . 7}$ & NC 7000 & $\mathbf{9 9 . 7}$ & Baytubes & $\mathbf{9 9 . 6}$ \\
\hline 6 & Baytubes & $\mathbf{9 9 . 8}$ & Baytubes & $\mathbf{9 9 . 8}$ & NC 7000 & $\mathbf{9 9 . 8}$ \\
\hline 7 & NC 7000 & $\mathbf{9 9 . 5}$ & Baytubes & $\mathbf{9 9 . 3}$ & Baytubes & $\mathbf{9 9 . 3}$ \\
\hline 8 & Baytubes & $\mathbf{9 9 . 4}$ & Baytubes & $\mathbf{9 9 . 3}$ & Baytubes & $\mathbf{9 9 . 3}$ \\
\hline 9 & Baytubes & $\mathbf{9 9 . 6}$ & Baytubes & $\mathbf{9 9 . 6}$ & Baytubes & $\mathbf{9 9 . 6}$ \\
\hline 10 & Baytubes & $\mathbf{9 9 . 8}$ & Baytubes & $\mathbf{9 9 . 8}$ & Baytubes & $\mathbf{9 9 . 8}$ \\
\hline
\end{tabular}

Table 3. Results from comparison of spectra from Baytubes $\mathrm{C} 150 \mathrm{HP}$ with references from the extended spectra database

\begin{tabular}{|c|c|c|c|c|c|c|}
\hline C-Tubes 200 & \multicolumn{2}{|c|}{ Hit 1 } & \multicolumn{2}{c|}{ Hit 2 } & \multicolumn{2}{c|}{ Hit 3 } \\
\hline Sample ID & Result: & $\%$ & Result: & $\%$ & Result: & $\%$ \\
\hline 1 & C-Tubes 200 & $\mathbf{9 9 . 3}$ & C-Tubes 200 & $\mathbf{9 9 . 3}$ & C-Tubes 200 & $\mathbf{9 9 . 2}$ \\
\hline 2 & Ahwahnee & $\mathbf{9 9 . 0}$ & C-Tubes 200 & $\mathbf{9 9 . 0}$ & C-Tubes 200 & $\mathbf{9 8 . 8}$ \\
\hline 3 & C-Tubes 200 & $\mathbf{9 9 . 7}$ & C-Tubes 200 & $\mathbf{9 9 . 7}$ & C-Tubes 200 & $\mathbf{9 9 . 7}$ \\
\hline 4 & C-Tubes 200 & $\mathbf{9 9 . 8}$ & C-Tubes 200 & $\mathbf{9 9 . 7}$ & C-Tubes 200 & $\mathbf{9 9 . 7}$ \\
\hline 5 & Ahwahnee & $\mathbf{9 9 . 5}$ & C-Tubes 200 & $\mathbf{9 9 . 3}$ & C-Tubes 200 & $\mathbf{9 9 . 2}$ \\
\hline 6 & C-Tubes 200 & $\mathbf{9 9 . 9}$ & C-Tubes 200 & $\mathbf{9 9 . 8}$ & Ahwahnee & $\mathbf{9 9 . 6}$ \\
\hline 7 & C-Tubes 200 & $\mathbf{9 9 . 6}$ & C-Tubes 200 & $\mathbf{9 9 . 5}$ & C-Tubes 200 & $\mathbf{9 9 . 4}$ \\
\hline 8 & C-Tubes 200 & $\mathbf{9 9 . 7}$ & C-Tubes 200 & $\mathbf{9 9 . 7}$ & C-Tubes 200 & $\mathbf{9 9 . 6}$ \\
\hline 9 & C-Tubes 200 & $\mathbf{9 9 . 7}$ & C-Tubes 200 & $\mathbf{9 9 . 5}$ & C-Tubes 200 & $\mathbf{9 9 . 5}$ \\
\hline 10 & C-Tubes 200 & $\mathbf{9 9 . 6}$ & C-Tubes 200 & $\mathbf{9 9 . 6}$ & C-Tubes 200 & $\mathbf{9 9 . 6}$ \\
\hline
\end{tabular}

Table 4. Results from comparison of spectra from $\mathrm{C}_{\text {Tube }} 200$ with references from the extended spectra database

It was possible to distinguish clearly between different mwCNT and other carbon-based sources (Table 1 to 4). All inspected mwCNT were detected as mwCNT and never as another carbon source. The hits from the database comparison selected the correct carbon specimen (mwCNT or other carbon source) with a very high likelihood. Even between different CNTmanufacturers, a differentiation is possible. As the Raman-signal increases with the amount of inspected material, a quantification based on the intensity of the Raman-signal will be possible. Furthermore, not only a physical fingerprint, but also a morphological information is gained from the investigated particles as a picture of its shape. Comparable spectra comparison results were obtained from the Raman-systems from PerkinElmer Inc. and Renishaw plc. 


\section{Summary}

The results show that Raman-spectroscopy coupled with a powerful data processing system can clearly distinguish between mwCNTs and other carbon containing sources and even a differentiation between different selected mwCNT manufacturers seems possible. Further more, it is possible to analyze the principal nature of particles deposited on a surface after impaction/sedimentation with Raman-spectroscopy. Therefore, an automated Raman-based detection of CNT-particles deposited on a suitable surface is feasible. This outcome will be investigated further in detail and the results will be presented at the $4 \mathrm{M}$ conference 2011.

\section{Acknowledgements}

The research leading to these results has received funding from the European Commission under grant agreement FP7-211464-2 (NANODEVICE) for the personal sampler and from the Bundesministerium für Bildung und Forschung BMBF under grant agreement 03FPF00024 (NanoSafety) for the Raman analysis. The application days at PerkinEImer LAS GmbH in Rodgau and Renishaw $\mathrm{GmbH}$ in Plietzhausen gave us the opportunity to demonstrate the possible qualitative CNT-determination on devices not available in our own laboratory.

\section{References}

[1]Elgrabi D. et al. Pulmonary toxicity comparison of raw and super-purified single-wall carbon nanotubes after intra-tracheal instillation in rats. INRS Occupational Heath Research Conference, Nancy, 2011

[2]Keller M., Neubauer N. \& Seipenbusch M. Monitoring method for nanofibers: Personal sampler and corresponding reading device. INRS Occupational Heath Research Conference, Nancy, 2011

[3]Gardiner D.J. Practical Raman spectroscopy. Springer, Berlin, 1989.

[4]Stigler S. M. Francis Galton's Account of the Invention of Correlation". Statistical Science 4 (2): 73-79, 1989. 